gave these values as rational fractions up to $m=12$, and Medhurst and Roberts [13] gave four more values, up to $m=16$.

Department of Engineering Mechanics

Virginia Polytechnic Institute

Blacksburg, Virginia

Department of Physics

University of Connecticut

Storrs, Connecticut

1. C.-B. LiNG, "Tables of values of the integrals $\int_{0}^{\infty}\left(x^{m} / \sinh ^{p} x\right) d x$ and $\int_{0}^{\infty}\left(x^{m} / \cosh ^{p} x\right) d x$," J. Math. and Phys., v. 31, 1952, pp. 58-62. MR 13, 690. (Two misprints are here noted. On p. 59, the subscript of $s^{\prime}$ in the last equation of (8) should be $m-2 p+2 r$. Again, on p. 60 , the exponent of 2 in the first equation of (9) should be $p+1$.)

2. C.-B. Ling \& C. W. NeLson, "On evaluation of Howland's integrals," Annals of Academia Sinica, no. 2, part 2, 1955, pp. 45-50.

3. C. W. NeLson, "A Fourier integral solution for the plane-stress problem of a circular ring with concentrated radial loads," $J$. Appl. Mech., v. 18, 1951, pp. 173-182. MR 12, 880.

4. C. W. Nelson, "New Tables of Howland's and related integrals," Math. Comp., v. 15, 1961, pp. 12-18. MR $22 * 10203$.

5. J. W. L. Glaisher, "Numerical values of the series $1-1 / 3^{n}+1 / 5^{n}-1 / 7^{n}+1 / 9^{n}-$ ...," Messenger of Math., v. 42, 1912, pp. 35-49.

6. J. W. L. Glaisher, "Tables of $1 \pm 2^{-n}+3^{-n} \pm 4^{-n}+$ etc. and $1+3^{-n}+5^{-n}+7^{-n}+$ etc. to 32 places of decimals," Quart. J. Pure and A ppl. Math., v. 45, 1914, pp. 141-152.

7. H. T. Davis, Tables of Higher Mathematical Functions, Vol. 2, Principia Press, Bloomington, Indiana, 1955.

8. J. Peters \& J. Stein, "Mathematical tables," Appendix of Peters' Ten-place Logarithmic Tables, Vol. 1, Ungar, New York, 1957.

9. A. H. R. GRIMSEY, "On the accumulation of chance effects and the Gaussian frequency distribution," Philos. Mag., v. 36, 1945, pp. 294-295. MR 1, 311.

10. L. S. GODDARD, "The accumulation of chance effects and the Gaussian frequency distribution," Philos. Mag., v. 36, 1945, pp. 428-433. MR 7, 311.

11. B. ButLer, "On the evaluation of $\int_{0}^{\infty}(\sin t / t)^{m} d t$ by the trapezoidal rule," Amer. Math. Monthly, v. 67, 1960, pp. 566-569.

12. K. Harumi, S. Katsura \& J. W. Wrench, Jr., "Values of $(2 / \pi) \int_{0}^{\infty}(\sin t / t)^{n} d t$," Math. Comp., v. 14, 1960, p. 379. MR 22 * 12737.

13. R. G. Medhurst \& J. H. Roberts, "Evaluation of the integral $(2 / \pi) \int_{0}^{\infty}(\sin t / t)^{m}$. $\cos$ bt dt," Math. Comp., v. 19, 1965, pp. 113-117.

\title{
Numerical Evaluation of the Elliptic Integral of the Third Kind
}

\section{By Charles H. Franke}

The purpose of this note is to point out a use of the addition formula for the elliptic integral of the third kind which significantly simplifies the numerical evaluation of the function.

The elliptic integral of the third kind may be defined by

$$
\Pi\left(n, k^{2}, \phi\right)=\int_{0}^{\phi} \frac{d \alpha}{\left(1+n \sin ^{2} \alpha\right)\left(1-k^{2} \sin ^{2} \alpha\right)^{1 / 2}} .
$$

Two standard power series are used to evaluate $\Pi\left(n, k^{2}, \phi\right)[2$, p. 5],

$$
\begin{aligned}
\Pi\left(n, k^{2}, \phi\right) & =\sum_{j=0}^{\infty} a(j) A(j) k^{2 j}, \\
a(j) & =\frac{(2 j) !}{2^{2}(j !)^{2}}, \quad A(j)=\int_{0}^{\phi} \frac{\sin ^{2 j} \alpha}{1+n \sin ^{2} \alpha} d \alpha,
\end{aligned}
$$

Received November 17. 1964. 


$$
\begin{aligned}
\Pi\left(n, k^{2}, \phi\right) & =\sum_{j=0}^{\infty} b(j) B(j)\left(1-k^{2}\right)^{j}, \\
b(j) & =\frac{(-1)^{j}(2 j) !}{2^{2 j}(j !)^{2}}, \quad B(j)=\int_{0}^{\phi} \frac{\sec \alpha \tan ^{2 j} \alpha}{1+n \sin ^{2} \alpha} d \alpha .
\end{aligned}
$$

Although the series (1) converges for $k^{2} \sin ^{2} \phi<1$, it is not useful for numerical computation for $k^{2} \sin ^{2} \phi$ near 1 . The series (2) converges only for $\left(1-k^{2}\right) \tan ^{2} \phi<1$, and is not useful for numerical computation for $\left(1-k^{2}\right) \tan ^{2} \phi$ near 1. Therefore, neither series can be used when $k^{2} \sin ^{2} \phi$ is near 1 unless $\left(1-k^{2}\right) \tan ^{2} \phi$ is significantly less than 1 (e.g., less than 0.7 ). A technique for evaluating $\Pi\left(n, k^{2}, \phi\right)$ for the range of the variables in which neither power series can be applied is through the application of the following addition formula $[3$, p. 13, $116.02,116.03]$,

$$
\begin{aligned}
& \Pi\left(n, k^{2}, \phi\right)=\Pi\left(n, k^{2}, \theta\right)+\Pi\left(n, k^{2}, \beta\right)-G, \\
& \phi=2 \arctan \left[\frac{\sin \theta\left(1-k^{2} \sin ^{2} \beta\right)^{1 / 2}+\sin \beta\left(1-k^{2} \sin ^{2} \theta\right)^{1 / 2}}{\cos \theta+\cos \beta}\right], \\
& A=\sin \theta \sin \beta \sin \phi\left|n(n+1)\left(n+k^{2}\right)\right|^{1 / 2} \text {, } \\
& B=1+n \sin ^{2} \phi-n \sin \theta \cos \phi\left(1-k^{2} \sin ^{2} \phi\right)^{1 / 2}, \\
& C=\left|n /(n+1)\left(n+k^{2}\right)\right|^{1 / 2} \text {, } \\
& G=C \arctan (A / B) \quad \text { if } 0<n \text { or } 0<k^{2}<-n<1 \text {, } \\
& =C \tanh ^{-1}(A / B) \quad \text { if } 0<-n<k^{2}<1 .
\end{aligned}
$$

A method in the literature for applying the addition formula is to fix $\beta$ at a convenient value, e.g., $45^{\circ}$, and solve (3) for $\theta$ [1]. If the solution is denoted by $\theta^{(1)}$, then $\theta^{(1)}<\phi$. This procedure may be repeated by using (3) with $\phi=\theta^{(1)}$ to determine $\theta^{(2)}$. In this way one may obtain a sequence $\theta^{(1)}, \cdots, \theta^{(j)}$ with $\theta^{(j)}$ small enough for efficient computation. The difficulty in this approach is that the number of iterations necessary to reduce $\theta^{(j)}$ below a given value increases as $k^{2}$ approaches 1 . For $\phi=90^{\circ}$, no fixed number of iterations will suffice to reduce $\theta^{(j)}$ below a fixed value for all $k^{2}<1$.

We will show that, if the addition formula is used as a "double-angle" formula, then one application is sufficient for computational purposes.

Taking $\beta=\theta$ in (3) and solving gives

$$
\sin ^{2} \theta=\frac{1-z}{k^{2}(1+\cos \phi)}
$$

where $z=\left(1-k^{2} \sin ^{2} \phi\right)^{1 / 2}$. Therefore,

$$
\tan ^{2} \theta=\frac{1-z}{k^{2}(1+\cos \phi)-1+z} .
$$

Rationalizing the numerator one obtains

$$
\tan ^{2} \theta=\frac{\sin ^{2} \phi}{\cos ^{2} \phi+\cos \phi+z(1+\cos \phi)} \leqq\left(1-k^{2}\right)^{-1 / 2} .
$$


Therefore, $\left(1-k^{2}\right) \tan ^{2} \theta \leqq\left(1-k^{2}\right)^{1 / 2}$ and power series (2) can be applied.

Based on the above, a ForTran IV program has been written using the following methods of evaluation.

(a) $k^{2} \leqq .64$. Power series (1).

(b) $\left(1-k^{2}\right) \tan ^{2} \phi \leqq .64$. Power series (2).

(c) $k^{2}>.64,\left(1-k^{2}\right) \tan ^{2} \phi>.64$. A single application of the double-angle formula followed by power series (2). (We note that, in the worst case, $\phi=90^{\circ}$, $k^{2}=.64+\delta$, the convergence of the power series used is like $.6^{j}$.)

The accuracy and timing of a program based on the method given above are dependent on the manner in which the computational details are handled. (E.g., the obvious recurrence formula for the $A(j)$ of $(1)$ cannot be used when $|n|$ is near zero.) The following remarks are meant to give some indication of the efficiency of a particular program.

The following chart shows the number of times an error of given magnitude occurred in the eighth significant digit in the computation of $\Pi\left(n, k^{2}, \phi\right)$ for $n=1,5$, $10,50,100 ; k^{2}=0.0,1.0(0.1) ; \phi=10^{\circ}, 90^{\circ}\left(10^{\circ}\right)$.

$\begin{array}{lrrrrrrr}\text { Error } & 0 & 1 & 2 & 3 & 4 & 5 & 6 \\ \text { Times Occurred } & 242 & 183 & 39 & 20 & 2 & 1 & 3\end{array}$

Near the pole $\Pi\left(n, 1,90^{\circ}\right)=\infty$, the relative error in the computation of $\Pi\left(n, k^{2}, 90^{\circ}\right)[\Pi(n, 1, \phi)]$ was about the same as the relative error in the computation of $1-k^{2}[(\pi / 2)-\phi]$. (A program could be written which accepts $1-k^{2}$ and $(\pi / 2)-\phi$ as alternate inputs. $)$

The average time required to evaluate $\Pi\left(n, k^{2}, \phi\right)$ for $n=1,100(3) ; k^{2}=0.0$, $1.0(0.1)$ and $\phi=1^{\circ}, 90^{\circ}\left(1^{\circ}\right)$ was 3.73 milliseconds. The time required to compute $\Pi\left(1 ., .64000001,90^{\circ}\right)$ was 10 milliseconds.

Bell Telephone Laboratories

Whippany, New Jersey

1. D. K. AI \& Z. L. HARRIson, A Computer Method for Calculating the Complete and Incomplete Elliptic Integrals of the Third Kind, Report AD-437-207, California Institute of Technologý, February, 1964.

2. V. M. Beljakov, R. I. Kracova \& M. G. Rappaport, Tables of Elliptic Integrals, Mathematical Tables of the Computing Center of the Academy of Sciences of the USSR, Vol. 2, Izdat. Akad. Nauk SSSR, Moscow, 1963. MR 28 *2259.

3. P. F. BYRD \& M. D. FRIEDMAN, Handbook of Elliptic Integrals for Engineers and Physicists, Die Grundlehren der mathmatischen Wissenschaften, Bd. LXVII, Springer, Berlin, 1954. MR 15, 702 . 